\title{
HABILIDAD DE CUIDADO EN CUIDADORES FAMILIARES DE PERSONAS CON ENFERMEDAD CRÓNICA Y SOBRECARGA PERCIBIDA
}

\author{
CARING ABILITY IN FAMILY CAREGIVERS OF PEOPLE WITH \\ CHRONIC DISEASE AND PERCEIVED OVERLOAD
}

\author{
Claudia Patricia Cantillo-Medina* \\ Claudia Andrea Ramírez-Perdomo * \\ Alix Yaneth Perdomo-Romero ${ }^{* * *}$
}

\begin{abstract}
RESUMEN
Objetivo: Analizar las características sociodemográficas del cuidador-persona con enfermedad crónica no transmisible y la asociación entre habilidad de cuidado, sobrecarga percibida por el cuidador y características sociodemográficas del cuidador-persona con enfermedad crónica no transmisible hospitalizada en una institución de alta complejidad. Material y método: Estudio analítico, descriptivo, correlacional, corte transversal, con 89 cuidadores. La información se recolectó mediante la utilización de los instrumentos: Ficha de caracterización de la díada, Inventario de Habilidad de Cuidado y la Escala de sobrecarga de Zarit. Resultados: Los cuidadores poseen bajo nivel de habilidades de cuidado $(84,3 \%)$ y refieren ausencia de sobrecarga $(70,8 \%)$. Se encontró asociación significativa, entre habilidad, sobrecarga y algunas características de la díada y del cuidado ( $<<0,05)$. Conclusión: Los resultados muestran afectación en la habilidad del cuidado y ausencia de sobrecarga, así como asociación entre habilidad y sobrecarga de los cuidadores, lo cual impacta la posibilidad de resolver las necesidades de cuidado de las personas dependientes.
\end{abstract}

Palabras clave: Cuidador familiar; Enfermedad crónica; Aptitud; Cuidado de Enfermería.

\begin{abstract}
Objective: To analyze the sociodemographic characteristics of the caregiver-person with a chronic noncommunicable disease and the association between caregiving ability, caregiver perceived overload, and the sociodemographic characteristics of the caregiver-person with a chronic non-communicable desease hospitalized in a high complexity center. Material and method: Analytical, descriptive, correlational, crosssectional study, carried out with 89 caregivers. Data were collected using the following instruments: Dyad Characterization Card, Caring Ability Inventory and Zarit Overload Scale. Results: Caregivers have a low level of caring abilities (84.3\%) and report absence of overload (70.8\%). A significant association was found between caring ability, overload and some characteristics of caregiving and dyads $\mathrm{p}<0.05$. Conclusion: Results show an impact on caring ability and absence of overload, as well as an association between ability and caregiver's overload, which affects the possibility of solving care needs of people.
\end{abstract}

Key words: Family caregiver; Chronic disease; Aptitude; Nursing care.

Fecha recepción: 13/04/18 Fecha aceptación:18/10/18

*Enfermera, Especialista en Enfermería Nefrológica y Urológica, Magíster en Enfermería. Docente Universidad Surcolombiana. Neiva-Huila-Colombia. Email: claudiacantillo1@hotmail.com. Autor de correspondencia.

${ }^{* *}$ Enfermera, Magíster en Enfermería con Énfasis en Gerencia en Servicios de Salud, Especialista en Enfermería en Cuidado Crítico Pontificia. Doctora $@$ en Enfermería, Universidad de Antioquia. Docente Universidad Surcolombiana. Neiva-Huila-Colombia. Email: clauram1@hotmail.com

${ }^{* * *}$ Enfermera, Magíster en Enfermería y en Educación. Docente Universidad Surcolombiana. Neiva-Huila-Colombia. Email: alixyaneth.perdomo@usco.edu.co 


\section{INTRODUCCIÓN}

El envejecimiento y las enfermedades no transmisibles de carácter crónico son algunos retos a los que se enfrentan actualmente los sistemas de salud del mundo, por la carga económica y social del cuidado de estas personas ${ }^{(1)}$. Lo anterior obliga a incrementar los rubros destinados a cubrir las incapacidades temporales o permanentes de quienes padecen estas enfermedades, así como también, a involucrar a familiares y cuidadores no profesionales a las tareas del cuidado(2).

Las enfermedades crónicas no transmisibles (ECNT) son aquellas de carácter no infeccioso y no transmisibles que se presentan en las personas ${ }^{(1)}$, son de larga duración y evolucionan lentamente; consideradas las principales causas de morbilidad y mortalidad en el mundo. Las cuatro principales ECNT son: cardiovasculares, cáncer, enfermedades respiratorias crónicas y la diabetes $^{(1)}$. Durante el año 2016 se calcula que se produjeron aproximadamente 41 millones de muertes, lo cual representó el 71\% de las defunciones a nivel global ${ }^{(2)}$, convirtiéndose de esta manera en una gran preocupación tanto para los países desarrollados como para los clasificados en vía de desarrollo.

Es así como el incremento de las enfermedades crónico-degenerativas y discapacitantes se traduce en una mayor demanda de cuidados para las personas que las padecen ${ }^{(3)}$, exigiendo en las familias cada vez más responsabilidades de atención, que imponen nuevas relaciones de reciprocidad y transferencias intergeneracionales con ayudas económicas y cuidados personales, convirtiéndose así en problemas de Salud Pública. Surge entonces el tema del cuidado como una prioridad de las políticas, así como la conformación de redes sociales que fortalezcan los factores protectores para una vejez digna, activa y saludable ${ }^{(4)}$.

La persona con enfermedad crónica por su estado de salud, alteración en su funcionalidad ${ }^{(5)}$ y dependencia, demanda mayor cantidad de cuidado en un tiempo prolongado o permanente, condición que requiere de servicios especializados, de alto costo y atención multidisciplinaria ${ }^{(6)}$; es aquí donde surge la necesidad de contar con un cuidador quien, al asumir este rol, debe hacer cambios en su rutina, requiere desarrollar habilidad para cuidar ${ }^{(7)}$ y prepararse para ejercer la labor de cuidar en el hogar ${ }^{(8)}$.

Un cuidador posee habilidad cuando entiende quién es la persona a cuidar, sus necesidades, fortalezas, debilidades y elementos para su bienestar, así como conocer sus capacidades y limitaciones, esa habilidad medida en conocimiento, paciencia y valor ${ }^{(9)}$.

Según Grazziano et al. ${ }^{(10)}$ son importantes los programas educativos como estrategias que contribuyan a apoyar el rol de cuidador, fortaleciendo el cuidado del paciente como de sí mismos, y de esta manera aportar en la prevención de las enfermedades, promoción de la salud y bienestar del cuidador.

La experiencia y habilidad de cuidado para los cuidadores ha sido evaluada en el contexto mundial $^{(11)}$, latinoamericano ${ }^{(12)}$ y en diferentes regiones de Colombia ${ }^{(13)}$, donde se ha reportado un nivel insuficiente en la habilidad ${ }^{(14)}$, además de percepción de sobrecarga producida por la labor de cuidar y atender las necesidades del otro, al incrementar las funciones propias de sus roles familiares y sociales ${ }^{(15)}$.

La valoración del nivel de habilidad, sobrecarga del cuidador y características de las personas enfermas permiten identificar las necesidades de cuidados en salud ${ }^{(14,16)}$, y plantear programas de apoyo y soporte con intervenciones específicas que proporcionen recursos que compensen los requerimientos para el cuidado de la díada cuidador-persona con ECNT.

Este panorama es un desafío para los sistemas de salud, los cuales deben pasar de un modelo curativo a la prestación de cuidados integrales, centrados en las necesidades de las personas mayores en quienes habrá un aumento de enfermedades que conllevan a secuelas incapacitantes, generando dependencia y necesidad de un cuidador con conocimientos, capacitación y apoyo adecuado que brinde cuidado en el hogar ${ }^{(17)}$. 
El papel del cuidador es sin lugar a dudas importante en el proceso de recuperación del enfermo, pero no es fácil para ninguno de los dos, ambos deben afrontar una serie de condiciones sociales que, sumadas a su estado emocional y al drama derivado de la enfermedad, los enfrentan a diversos obstáculos; es ahí donde la preparación del cuidador juega un papel primordial, al recaer en él la confianza, tranquilidad y seguridad que el enfermo temporalmente ha perdido y seguramente recuperará a medida que el cuidador vaya asumiendo su rol ${ }^{(18)}$.

Esa persona que desempeña la importante labor de cuidar y atender las necesidades de personas desde su casa y a partir de los recursos disponibles, sobrecarga las funciones propias de su rol familiar y social, adaptándose a nuevas condiciones para mejorar la salud y bienestar de quien cuida. El cuidado se convierte en un batallar diario contra la enfermedad, las tareas monótonas y repetitivas producen sensación de falta de control sobre el resultado de dicha labor hasta agotar las reservas psicofísicas del cuidador, produciendo sobrecarga y afectando la calidad de vida ${ }^{(18)}$. Teniendo en cuenta la dura y compleja labor que representa el papel del cuidador, este estudio busca analizar las características sociodemográficas del cuidador-persona con enfermedad crónica no transmisible y la asociación entre habilidad de cuidado, sobrecarga percibida por el cuidador y características sociodemográficas del cuidador-persona con enfermedad crónica no transmisible hospitalizada en una institución de alta complejidad en la ciudad de Neiva-Colombia.

\section{MATERIAL Y MÉTODO}

Estudio analítico, descriptivo, correlacional, de corte transversal.

Muestreo no aleatorizado por conveniencia. La muestra estuvo conformada por $n=89$ personas con ECNT, a quienes se les evaluó las características sociodemográficas y el grado de dependen- cia y $n=89$ cuidadores de personas con diferentes ECNT hospitalizadas en una institución de alta complejidad, a quienes se les aplicaron los instrumentos que se describen más adelante. El tamaño de la muestra se determinó con el número de personas hospitalizadas durante los meses de septiembre y octubre de 2017.

Los criterios de inclusión propuestos para el grupo de cuidadores fueron: mayor de 18 años, cuidador de persona con enfermedad crónica hospitalizada, diligenciamiento y autorización del consentimiento informado.

Los participantes fueron contactados en el servicio de hospitalización, se les dio a conocer el objetivo de la investigación y previa firma del consentimiento informado se recolecto la información, lo cual fue realizado por las investigadoras y los auxiliares de investigación.

Los instrumentos utilizados fueron:

a) Ficha de caracterización de la díada persona con ECNT - cuidador familiar del Grupo de investigación Cuidado de Enfermería al paciente crónico (GCPC-UN-D) ${ }^{(19)}$ organizada en tres dimensiones: identificación de condiciones del paciente y del cuidador y su perfil sociodemográfico, percepción de carga y de apoyo, medios de información y comunicación; cuenta con validez facial y de contenido para América Latina.

b) Pulses, escala de valoración funcional creada y empleada por Moskowitz en $1957^{(20)}$, se refiere a seis funciones $\mathrm{P}=$ estabilidad de la patología o condición física, $\mathrm{U}=$ utilización de miembros superiores, $\mathrm{L}=$ locomoción o función de los miembros inferiores, $\mathrm{S}=$ función sensorial, $\mathrm{E}=$ eliminación o control de esfínteres, $\mathrm{S}=$ capacidad de socializar. Evalúa no solo la capacidad funcional aislada sino la capacidad para realizar actividades de la vida diaria.

c) SPMSQ-PFEIFFER, Cuestionario corto portátil de valoración de estado mental. Es un test de detección de deterioro cognitivo, que valora 4 parámetros: memoria a corto y largo plazo, orien- 
tación, información sobre hechos cotidianos y capacidad de cálculo ${ }^{(21)}$.

d) El Inventario de Habilidad de Cuidado (CAI por sus siglas en inglés) de Nkongho ${ }^{(9)}$, que consta de 37 ítems, con Alfa de Cronbach de 0,89 y estabilidad de $\mathrm{r}=0,80$ en Colombia $^{(22)}$; los puntajes más altos indican mayor habilidad para cuidar de manera general y por las subescalas que responden a 3 factores asociados con el entendimiento del propio ser y de otros: conocimiento, valor y paciencia, los cuales se clasifican en bajo (203 o menos), medio $(203,1-220,2)$ y alto $(220,3 \mathrm{o}$ más).

e) La Escala de sobrecarga de Zarit ${ }^{(23)}$ evalúa varios factores: el impacto del cuidado, las relaciones interpersonales y las expectativas de autoeficacia. Contiene 22 preguntas tipo Likert con rango de respuesta de 0 a 4 (nunca, casi nunca, a veces, muchas veces, siempre). Cuando el rango de respuestas es menor de 46 indica no sobrecarga, de 46 a 56 sobrecarga leve, mayor de 56 sobrecarga intensa, con Alfa de Cronbach de 0,86 para el contexto colombiano. Todos los instrumentos respectivamente fueron diligenciados en línea utilizando la aplicación de Formularios de Google Drive.

Esta investigación fue aprobada por el Comité de Ética y Bioética de la Universidad Surcolombiana acta No 002 de 2017, teniendo en cuenta los principios bioéticos: respeto a la dignidad humana, privacidad, libertad de expresión y sentimientos, confidencialidad y reciprocidad.

El análisis de la información se realizó mediante el programa estadístico IBM SPSS ${ }^{\circledR}$, en su versión № 23. Se emplearon frecuencias absolutas y relativas para las variables cualitativas y medidas de tendencia central y de dispersión para las variables cuantitativas. Adicionalmente se realizó análisis bivariado para correlacionar y/o asociar variables como habilidad del cuidado, sobrecarga percibida y características de la díada. Se aplicaron diferentes pruebas de confianza estadística como Rho de Spearman y U de Mann Whitney.

\section{RESULTADOS}

Los participantes pertenecientes al sexo femenino, persona cuidada y cuidadores 60,7 y $75,3 \%$, respectivamente. El promedio de edad fue de 56,2 años (DE 23,7) con una mediana de 65,2 (Mín. 1; Máx. 92) en las personas con ECNT y de 42,1 años (DE 16) con una mediana de 41,5 (Mín. 18; Máx. 72) en los cuidadores.

En cuanto al nivel funcional, las personas con ECNT (medido por la escala PULSES) se encuentran en alto nivel de dependencia $68,6 \%$; con función mental intacta el 76,4\% valorado con el test SPMSQ; los cuidadores con un grado de escolaridad y nivel socioeconómico bajo, dedicados al hogar, el estado civil predominante fue casados y el cuidador principalmente el hijo (Tabla 1).

El 39,3\% de las personas enfermas perciben ser una carga baja para sus cuidadores; en la familia existe una responsabilidad compartida del cuidado 59,6\%; llevan entre 0 a 6 meses cumpliendo la labor de cuidado el $69,7 \%$ y con una dedicación al cuidado mayor a $20 \mathrm{~h}$ diarias $44,9 \%$ (Tabla 1).

En cuanto a las categorías y la calificación global de las habilidades del cuidador, la mayoría de la población se clasificó en un nivel bajo (Tabla 2).

Con referencia a la valoración de la sobrecarga percibida, el porcentaje superior $(70,8 \%)$ refirió ausencia de la misma, mientras que el 13,5\% la calificó como leve y el 15,7\% como intensa.

Se observan correlaciones bajas y estadísticamente significativas entre las características de la díada con la habilidad del cuidado y sobrecarga $(p \leq 0,05)$; correlaciones moderadas entre la sobrecarga percibida y sobrecarga $(\mathrm{p} \leq 0,00), y$ entre las categorías de la habilidad del cuidado $(\mathrm{p} \leq 0,00)$ (Tabla 3$)$.

Se realizó correlación entre las variables sociodemográficas del cuidador-persona cuidada y la habilidad de cuidado, pero no se encontraron asociaciones significativas.

En relación al nivel de percepción de la sobrecarga del cuidador en asociación con el sexo, se 
identificó relación estadísticamente significativa, nivel de percepción de la sobrecarga del cuidador que indica que existe mayor sobrecarga intensa con otras variables sociodemográficas del cuidaen los hombres $(\mathrm{p}=0,010<0,05)$ (Tabla 4$)$. No se dor-persona.

encontraron asociaciones significativas entre el

Tabla 1. Características de la díada (persona con ECNT - cuidador familiar) (n=89).

\begin{tabular}{|c|c|c|c|c|c|}
\hline \multirow[t]{2}{*}{ Variable } & & \multicolumn{2}{|c|}{ Persona con ECNT } & \multicolumn{2}{|c|}{ Cuidador } \\
\hline & & $\mathrm{N}$ & $\%$ & $\mathrm{n}$ & $\%$ \\
\hline \multirow[t]{2}{*}{ Sexo } & Masculino & 35 & 39,3 & 22 & 24,7 \\
\hline & Femenino & 54 & 60,7 & 67 & 75,3 \\
\hline Edad & Media (DE) & \multicolumn{2}{|c|}{$56,2(23,7)$} & \multicolumn{2}{|c|}{$42,1(16)$} \\
\hline \multirow{5}{*}{ Rangos de edad } & $<18$ años & 10 & 11,2 & 1 & 1,2 \\
\hline & 18 a 35 años & 14 & 15,7 & 36 & 41,9 \\
\hline & 36 a 59 años & 16 & 18 & 35 & 40,7 \\
\hline & 60 a 75 años & 30 & 33,7 & 14 & 16,3 \\
\hline & $>75$ años & 19 & 21,3 & 0 & 0 \\
\hline \multirow[t]{3}{*}{ PULSES (nivel de dependencia) } & 6-8 (bajo) & 18 & 20,2 & \multirow{3}{*}{\multicolumn{2}{|c|}{ NA }} \\
\hline & 9-11 (medio) & 10 & 11,2 & & \\
\hline & 12-24 (alto) & 61 & 68,6 & & \\
\hline \multirow[t]{4}{*}{ Test SPMSQ (función mental) } & $0-2$ (intacta) & 68 & 76,4 & \multirow{4}{*}{\multicolumn{2}{|c|}{ NA }} \\
\hline & 3-4 (alteración mínima) & 5 & 5,6 & & \\
\hline & 5-7 (alteración moderada) & 11 & 12,4 & & \\
\hline & 8-10 (alteración severa) & 5 & 5,6 & & \\
\hline \multirow[t]{9}{*}{ Grado máximo de escolaridad } & Analfabeta & 9 & 10,1 & 2 & 2,3 \\
\hline & Primaria incompleta & 20 & 22,5 & 18 & 20,9 \\
\hline & Primaria completa & 25 & 28,1 & 17 & 19,8 \\
\hline & Secundaria incompleta & 7 & 7,9 & 10 & 11,6 \\
\hline & Secundaria completa & 17 & 19,1 & 23 & 26,7 \\
\hline & Técnico/Tecnólogo & 3 & 3,4 & 9 & 10,5 \\
\hline & Universidad incompleta & 1 & 1,1 & 1 & 1,2 \\
\hline & Universidad completa & 7 & 7,9 & 6 & 7 \\
\hline & Postgrados & 0 & 0 & 0 & 0 \\
\hline \multirow[t]{4}{*}{ Ocupación } & Empleado(a) & 10 & 11,2 & 11 & 12,4 \\
\hline & Estudiante & 10 & 11,2 & 6 & 6,7 \\
\hline & Hogar & 52 & 58,4 & 50 & 56,2 \\
\hline & Trabajo independiente & 17 & 19,2 & 22 & 24,7 \\
\hline \multirow[t]{3}{*}{ Nivel socioeconómico. } & Bajo & 83 & 93,3 & 78 & 87,6 \\
\hline & Medio & 5 & 5,6 & 10 & 11,2 \\
\hline & Alto & 1 & 1,1 & 1 & 1,1 \\
\hline
\end{tabular}


CIENCIA y ENFERMERIA (2018) 24: 16

Continuación Tabla 1.

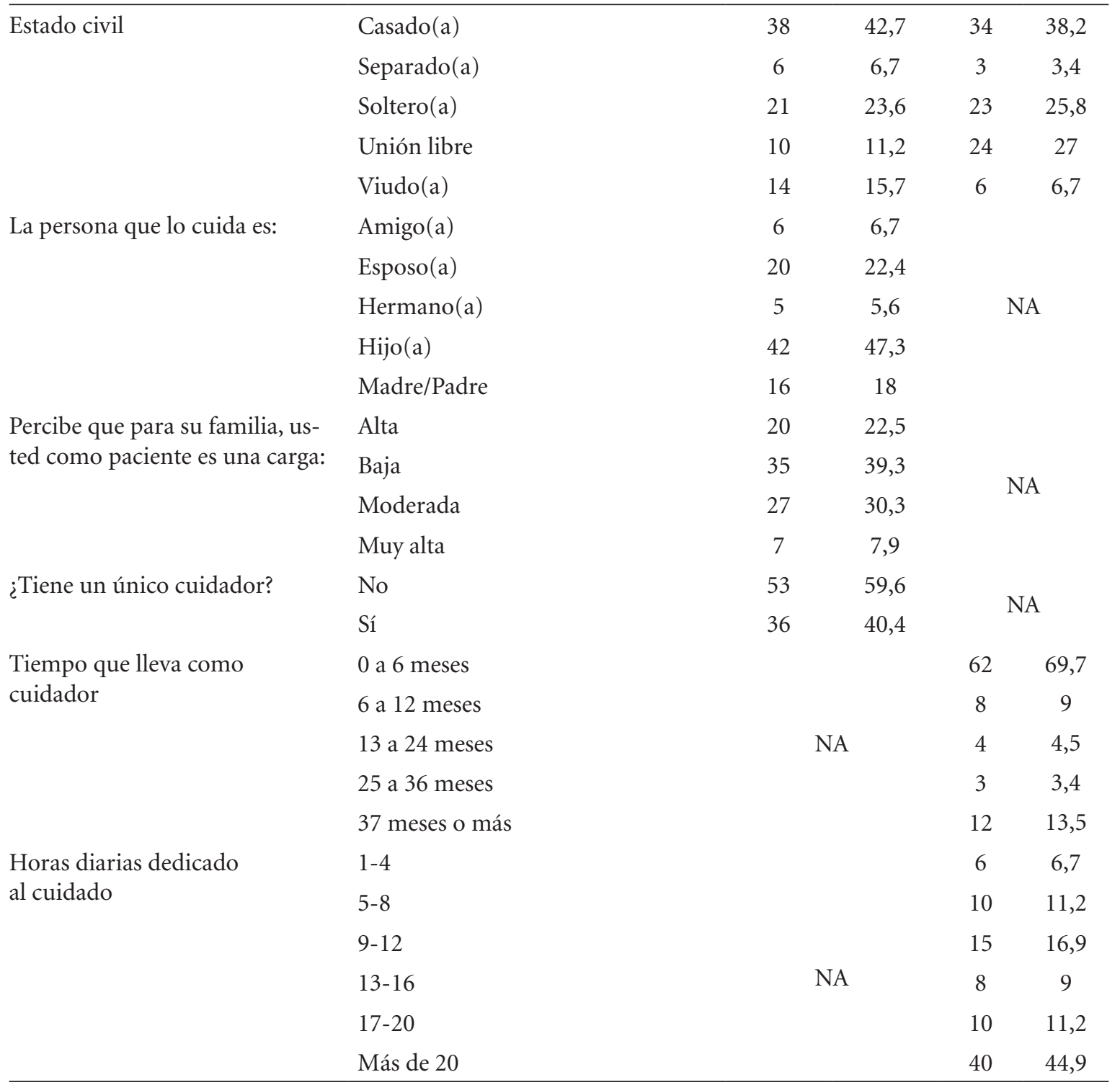


Tabla 2. Resultados de la medición del Inventario habilidad de cuidado, CAI ( $\mathrm{n}=89$ ).

\begin{tabular}{lccc}
\hline Categoría & Nivel & N & $\%$ \\
\hline Conocimiento & Baja & 44 & 49,4 \\
& Media & 16 & 18 \\
Paciencia & Alta & 29 & 32,6 \\
& Baja & 72 & 80,9 \\
Valor & Media & 17 & 19,1 \\
& Alta & 0 & 0 \\
& Baja & 71 & 79,8 \\
Habilidad Global & Media & 12 & 13,5 \\
& Alta & 6 & 6,7 \\
& Baja & 75 & 84,3 \\
& Media & 14 & 15,7 \\
\hline
\end{tabular}

Tabla 3. Correlaciones entre características de la díada, habilidades y sobrecarga del cuidador $(\mathrm{n}=89)$.

\begin{tabular}{|c|c|c|c|c|c|}
\hline \multirow[b]{2}{*}{ Variables } & \multicolumn{4}{|c|}{ Habilidad } & \multirow[t]{2}{*}{ Sobrecarga } \\
\hline & Conocimiento & Paciencia & Valor & $\begin{array}{l}\text { Habilidad } \\
\text { Global }\end{array}$ & \\
\hline PULSES-persona con ECNT & $-0,059$ & $-0,027$ & $-0,277$ & $-0,111$ & 0,048 \\
\hline Test SPMSQ-persona con ECNT & $0,255^{\star}$ & 0,051 & 0,137 & $-0,093$ & 0,123 \\
\hline Grado máximo de escolaridad-Paciente & $0,213^{*}$ & $-0,047$ & 0,033 & $-0,110$ & $-0,325^{\star *}$ \\
\hline Grado máximo de escolaridad-Cuidador & 0,111 & 0,072 & $-0,041$ & $-0,108$ & $-0,294$ \\
\hline Tiempo que lleva con la enfermedad crónica & $-0,122$ & $-0,028$ & 0,091 & $-0,059$ & $0,286^{\star *}$ \\
\hline Nivel socioeconómico - cuidador & 0,038 & $-0,086$ & $-0,100$ & $-0,058$ & $-0,238$ \\
\hline $\begin{array}{l}\text { Globalmente que grado de carga experimenta } \\
\text { por el hecho de cuidar a su familiar }\end{array}$ & $-0,039$ & 0,174 & $0,246^{*}$ & $0,234^{*}$ & $0,415^{\star *}$ \\
\hline
\end{tabular}

${ }^{*}$ La correlación es significativa en el nivel $\mathrm{p}<0,05$ (bilateral)

${ }^{*}$ La correlación es significativa en el nivel $\mathrm{p}<0,01$ (bilateral)

Tabla 4. Sobrecarga del cuidador en asociación con el género $(n=89)$.

\begin{tabular}{|c|c|c|c|c|c|c|c|}
\hline \multirow{3}{*}{ Sexo } & \multicolumn{6}{|c|}{ Sobrecarga } & \multirow{3}{*}{$\mathrm{p}$} \\
\hline & \multicolumn{2}{|c|}{ Ausencia } & \multicolumn{2}{|c|}{ Leve } & \multicolumn{2}{|c|}{ Intensa } & \\
\hline & $\mathrm{n}$ & $\%$ & $\mathrm{~N}$ & $\%$ & $\mathrm{n}$ & $\%$ & \\
\hline Masculino & 11 & 50 & 4 & 18,2 & 7 & 31,8 & \multirow{2}{*}{$0,010^{*}$} \\
\hline Femenino & 52 & 77,6 & 8 & 11,9 & 7 & 10,4 & \\
\hline
\end{tabular}

${ }^{\star} \mathrm{U}$ de Mann Whitney 


\section{DISCUSIÓN}

Los resultados de la caracterización de la díada cuidador-persona con enfermedad crónica no transmisible coinciden con lo descrito en la literatura, puesto que el rol de cuidadores lo asumen mayoritariamente las mujeres ${ }^{(14-16,18,24)}$ cuya edad se encontró por encima de 36 años (media 42,1), etapa de la vida considerada productiva laboralmente, situación que las ubica en condición de vulnerabilidad, debido a los riesgos que enfrentan al asumir gran cantidad de roles ${ }^{(19)}$ en relación a los otros miembros de la familia y de la sociedad.

En cuanto a la variable edad de las personas con ECNT, sobresale el rango de 60 a 75 años, quienes presentan un alto nivel de dependencia, condición similar reportada en diversos estu$\operatorname{dios}^{(5,25,26)}$; con una función mental intacta. La mayoría personas con bajo nivel educativo, circunstancia que favorece un control inadecuado de patologías precursoras de enfermedades crónicas $^{(16,24)}$, por falta de conocimiento de la enfermedad y su manejo. En la medida en que las personas tienen mayor comprensión de su situación se espera un aumento de la capacidad para cuidar de sí mismas ${ }^{(27)}$; la mayoría de las díadas con un nivel socioeconómico bajo y dedicación al ho$\operatorname{gar}^{(16)}$, condiciones que favorecen la invisibilidad de la labor del cuidado de la persona en situación de cronicidad, se incrementa la vulnerabilidad de las familias pues las empobrecen ${ }^{(28)}$, al asumir requerimientos que no son cubiertos por el sistema de salud ${ }^{(29)}$.

En relación al estado civil casados y el nexo con la persona cuidada: hijo, estos aspectos son coherentes con el ciclo vital y familiar de los participantes $^{(30)}$, en el cual los hijos son quienes asumen el cuidado de sus padres, contexto fundamental en el proceso de afrontamiento a la situación de salud, porque un vínculo de parentesco o afectivo-amoroso hace la diferencia entre el cuidado del familiar y el profesional ${ }^{(31)}$, además, puede ser un factor que influye en la calidad de vida de los cuidadores, pues el contar con una pa- reja estable puede influir en el apoyo percibido, afrontamiento y relevo ${ }^{(32)}$.

Tiempo que lleva como cuidador de 0 a 6 meses y dedicación mayor a 20 horas, elementos significativos en la experiencia del cuidado; en la medida que las personas comparten estos momentos, es tan fuerte el sentimiento experimentado que asumen conciencia del cuidado ${ }^{(33)}$; la mayoría de los cuidadores tienen apoyo de otro cuidador, aspecto protector para la no percepción de sobrecarga, hallazgos concordantes con los resultados de las investigaciones de Perdomo et al. ${ }^{(15)}$ y Ramírez et al. ${ }^{(18)}$.

En cuanto a las habilidades del cuidador, la mayoría de la población puntuó en nivel bajo para la calificación global y las categorías de conocimiento, paciencia y valor, similar con los hallazgos reportados por Benítez y Carreño ${ }^{(14)}$; estos resultados demuestran habilidad inapropiada para resolver las necesidades de atención de las personas dependientes de cuidado.

Alto nivel de dependencia de la persona cuidada, con menor habilidad valor, son aspectos que pueden reflejar la incertidumbre del cuidador frente al reto de asumir el cuidado en la cronicidad. La función mental intacta con menor habilidad de conocimiento son condiciones que favorecen la autonomía de la persona cuidada y evitan la sobrecarga del cuidador.

Además correlación positiva entre escolaridad de la persona con ECNT con habilidad conocimiento, pues la educación facilita el desarrollo de habilidades de cuidado, datos coherentes con los reportados por Torres et al. ${ }^{(16)}$; y presentaron correlación moderada entre las categorías de habilidad del cuidado: conocimiento con paciencia, paciencia con habilidad global y valor con habilidad global. Lo anterior indica que el cuidador desarrolla las tres competencias básicas de habilidad de cuidado, el conocimiento de sí mismo y su receptor de cuidados, con la paciencia que permite encontrar un sentido al rol de cuidador y el valor para tomar decisiones informadas y acceder a redes de apoyo social ${ }^{(34)}$.

Se encontró correlación positiva entre la carga global experimentada por la labor del cuidado 
con habilidad global y valor; el hecho de cuidar al familiar potencia la fuerza interior del cuidador, hallazgos que discrepan con los planteados por Carreño y Chaparro ${ }^{(35)}$; además, correlación positiva entre el tiempo que lleva con la enfermedad crónica y la sobrecarga experimentada, hallazgos coherentes con los resultados descritos por los autores anteriormente mencionados ${ }^{(35)}$.

Asimismo, a mayor grado de escolaridad y nivel socioeconómico de la díada, menor sobrecarga; correlación moderada entre nivel de percepción de la carga y sobrecarga, similar a lo reportado por Torres et al. ${ }^{(16)}$; se encontró correlación entre las variables sexo y sobrecarga, donde el mayor porcentaje de sobrecarga intensa estuvo presente en los cuidadores hombres, aná$\operatorname{logo}$ a lo reportado por Carreño y Chaparro ${ }^{(35)}$; situación interesante de profundizar en futuros procesos investigativos.

De acuerdo con los resultados encontrados se evidencia un nivel inadecuado de habilidad en los cuidadores, tanto en las categorías de conocimiento, valor y paciencia como en la medición global; los hallazgos reflejan que las variables de las personas con ECNT como nivel funcional de baja dependencia, función mental alterada y mejor nivel de escolaridad, potencian las habilidades del cuidado en todas las categorías; además un mejor nivel educativo y socioeconómico de la díada favorece la ausencia de sobrecarga, datos concordantes con lo referido por Torres et al. ${ }^{(16)}$.

Estos aspectos descritos anteriormente son útiles para avanzar en la cualificación de la atención de los profesionales de la salud, en especial Enfermería, quien está llamada a desarrollar relaciones de cuidado que abarquen la díada, diseñar estrategias de intervención participativa que sirvan para empoderar y fortalecer el grado de habilidad de cuidado, teniendo en cuenta que la condición de la persona que vive con ECNT convierte al cuidador en sujeto-objeto de cuidado, quien requiere de entrenamiento, acompañamiento, seguimiento y evaluación continua, para el control de las enfermedades crónicas ${ }^{(24)}$, además de reducir complicaciones y mejorar la calidad de vida.
Es importante continuar con el desarrollo de programas de soporte a los cuidadores, enfocados al perfil de los participantes, sus necesidades y planificación de cuidados de Enfermería, teniendo en cuenta los requerimientos individuales, las diversas dimensiones del ser humano y sus contextos, los cuales comprenden "La dimensión subjetiva y experiencial, que resultan determinantes en el afrontamiento de los procesos cróni$\cos ^{\prime \prime(36)}$, para brindar un cuidado eficaz y efectivo y así aportar a la disminución de la sobrecarga y mejoramiento de la calidad de vida de estos héroes, que han asumido la labor del cuidado de sus seres queridos.

\section{CONCLUSIÓN}

La mayoría de los cuidadores no perciben sobrecarga; se evidencia relación entre la habilidad del cuidado, sobrecarga y algunas características sociodemográficas de los cuidadores y de las personas con ECNT, por lo cual se ratifica la importancia de desarrollar estrategias participativas que impacten en el empoderamiento de las habilidades del cuidado, que fomenten la adherencia a los cuidados, eviten complicaciones y logren mejores desenlaces en salud.

Teniendo en cuenta que la población es cada vez más adulta y vulnerable, es fundamental mejorar el nivel educativo de la díada para fortalecer su conocimiento, que los lleven a asumir cambios en el comportamiento y conductas permanentes para mantener su grado de funcionalidad, actividad mental y autocuidado; además de soporte psicosocial que impacte de manera positiva su calidad de vida.

La limitación de esta investigación fue el empleo de un muestreo por conveniencia, situación que no hace posible la generalización de los resultados de la investigación. Se resalta la importancia de continuar desarrollando investigaciones en los aspectos de habilidad de cuidado y sobrecarga del cuidador, con el fin de aportar al bienestar de esta población. 
Agradecimientos: Se agradece a la Vicerrectoría de Investigación y Proyección Social, estudiantes del programa de Enfermería y Artes, al estadístico de la Unidad de Investigación de la Universidad Surcolombiana, así como a los cuidadores y personas con ECNT, y a las enfermeras del Hospital Universitario Hernando Moncaleano Perdomo de Neiva. Así como al Grupo de Cuidado al Paciente Crónico de la Universidad Nacional de Colombia.

\section{REFERENCIAS}

1. Kim HC, Oh SM. Noncommunicable Diseases: Current Status of Major Modifiable Risk Factors in Korea. J Prev Med Public Health. 2013; 46(4): 165-172.

2. World Health Organization. World health statistics 2018: monitoring health for the SDGs, sustainable development goals [Internet]. Geneva: World Health Organization; 2018 [cited 2017 Oct 08]. Available from: http://apps.who.int/iris/bitstream/handle/10 665/272596/9789241565585-eng.pdf

3. Comisión Económica para América Latina y el Caribe (CEPAL). Panorama Social de América Latina 2016 [Internet]. Santiago, Chile: CEPAL; 2017 [citado 08 Oct 2017]. Disponible en: https://repositorio.cepal.org/ bitstream/handle/11362/41598/4/S1700567_ es.pdf

4. Ministerio de Salud y Protección Social. Envejecimiento Demográfico. Colombia 19512020 Dinámica Demográfica y Estructuras Poblacionales [Internet]. Bogotá, D.C.: MINSALUD; 2013 [citado 08 Oct 2017]. Disponible en: https://www.minsalud.gov.co/ sites/rid/Lists/BibliotecaDigital/RIDE/DE/ PS/Envejecimiento-demografico-Colombia-1951-2020.pdf

5. Villarreal G, Month E. Condición sociofamiliar, asistencial y de funcionalidad del adulto mayor de 65 años en dos comunas de Sincelejo (Colombia). Salud Uninorte. 2012; 28(1): 75-87.
6. Arango DC, Peláez E. Envejecimiento poblacional en el siglo XXI: Oportunidades, retos y preocupaciones. Salud Uninorte. 2012; 28(2): 335-48.

7. Achury DM, Castaño H, Gómez L, Guevara N. Calidad de vida de los cuidadores de pacientes con enfermedades crónicas con parcial dependencia. Investigación en Enfermería: Imagen y Desarrollo. 2011; 13(1): 27-46.

8. Carrillo GM, Barreto RV, Arboleda LB, Gutiérrez OA, Melo BG, Ortiz VT. Competencia para cuidar en el hogar de personas con enfermedad crónica y sus cuidadores en Colombia. Rev. Fac. Med. 2015; 63(4): 668-675.

9. Nkongho N. The Caring Ability Inventory. En: Strickland OL,Waltz CF. Measurement of Nursing Outcomes: Self Care and Coping. New York: Springer Publishing Company; 1996.

10. Grazziano E, dos Reis E, Leda S, Dias Lopes L. Impact by care guidelines and perception of the quality of life in caregivers of the elderly. J Nurs UFPE on line. [Internet]. 2014. [citado 2017 Oct 08]; 8(8): 2823-32. Available from: https://periodicos.ufpe.br/revistas/revistaenfermagem/article/view/990

11. Martínez Marcos M, De La Cuesta Benjumea C. The experience of women care cargivers with chronic conditions of dependent relatives. Aten Primaria. 2016; 48(2): 77-84.

12. Eterovic C, Mendoza Parra S, Sáez Carrillo K. Habilidad de cuidado y nivel de sobrecarga en cuidadoras/es informales de personas dependientes. Enferm. glob. [Internet]. 2015 Abr [citado 13 Oct 2018]; 14(2): 235-48. Disponible en: https://revistas.um.es/eglobal/article/view/198121

13. Marín G, Alarcón M, Ramírez M. Habilidad de cuidado del cuidador informal del niño con leucemia. Enlaces Académicos. 2011; 4(1): 38-43.

14. Benítez M, Carreño S. Habilidad de cuidado y sobrecarga en cuidadores familiares de personas con cáncer. RFS Revista Facultad De Salud [Internet]. 2015 [citado 10 Dic 2017]; 7(2): 9-14. Disponible en: https://www.journalusco. edu.co/index.php/rfs/article/view/949 
15. Perdomo AY, Ramírez CA. Percepción de calidad de vida en cuidadores de pacientes con demencia. Rev Cient Soc Esp Enferm Neurol. 2017; 46: 26-31.

16. Torres X, Carreño S, Chaparro L. Factores que influencian la habilidad y sobrecarga del cuidador familiar del enfermo crónico. Rev. Univ. Ind. Santander Salud. 2017; 49(2): 33038.

17. Jofré V, Sanhueza O. Evaluación de la sobrecarga de Cuidadoras/es Informales. Cienc. enferm. 2010; XVI(3): 111-120.

18. Ramírez CA, Salazar Y, Perdomo AY. Quality of life of caregivers of persons with neurological disorder sequels. Rev Cient Soc Esp Enferm Neurol. 2017; 45: 9-16.

19. Chaparro L, Sánchez B, Carrillo G. Encuesta de caracterización del cuidado de la díada cuidador familiar - persona con enfermedad crónica. Rev Cien Cuidad. 2014; 11(2):31-45.

20. Bermejo Pareja F, Porta-Etessam J, Díaz Guzmán J, Martínez-Martín P. Más de cien escalas en neurología. $2^{\text {da }}$ ed. Toledo: Aula Médica; 2008.376 p.

21. Martínez de la Iglesia J, Dueñas R, Onís MC, Aguado C, Albert C, Luque R. Adaptación y validación al castellano del cuestionario de Pfeiffer (SPMSQ) para detectar la existencia de deterioro cognitivo en personas mayores de 65 años. Medicina Clínica 2001; 117(4): 129-134.

22. Barrera L, Sánchez B, Carrillo G, Chaparro L, Carreño S. Validación semántica y prueba de estabilidad del "Inventario de habilidad de cuidado" versión en español. Actualizaciones en Enfermería. 2015; 18(1): 8-14.

23. Barreto R, Campos M, Carrillo G, Coral R, Chaparro L, Durán et al. Entrevista Percepción de Carga del Cuidado de Zarit: pruebas psicométricas para Colombia. Aquichan. 2015; 15(3): 368-80.

24. Espinoza K, Jofre V. Sobrecarga, apoyo social y autocuidado en cuidadores informales. Cienc. enferm. 2012; XVIII(2): 23-30.

25. Martínez ME, Plazas M, Barajas GP, Bravo AM, González C, Rodríguez A et al. Factores de riesgo para enfermedad renal crónica en pacientes que asisten a consulta de medicina interna. Acta Med Colomb. 2013; 38(4): 22832.

26. Cantillo CP, De Jesús Blanco J. Agencia de Autocuidado de la persona con Enfermedad Renal Crónica en diálisis. Enferm Nefrol. 2017; 20(4): 330-41.

27. Pérez C, Riquelme G, Scharager J, Armijo I. Relación entre calidad de vida y representación de enfermedad en personas con enfermedad renal crónica terminal en tratamiento con hemodiálisis. Enferm Nefrol. 2015; 18(2): 89-96.

28. Bona K, Dussel V, Orellana L, Kang T, Geyer $\mathrm{R}$, Feudtner $\mathrm{C}$ et al. Economic impact of advanced pediatric cancer on families. J Pain Symptom Manage. 2014; 47(3): 594-603.

29. Montoya A, Montoya I, Ocampo J, SánchezHerrera B, Chaparro-Díaz L, Sánchez B. Diseño y validación de la encuesta costo financiero del cuidado de la enfermedad crónica. Salud Uninorte. 2017; 33(3): 355-62.

30. Semenova N, Zapata JJ, Messager T. Conceptualización de ciclo vital familiar: una mirada a la producción durante el periodo comprendido entre los años 2002 a 2015. CES Psicol [Internet]. 2015 Jul-Dic [citado 08 Oct 2017]; 8(2): 103-21. Disponible en: http:// revistas.ces.edu.co/index.php/psicologia/article/view/3555

31. De la Cuesta C. Cuidado Familiar en Condiciones Crónicas: Una Aproximación a la Literatura. Texto contexto - enferm. 2004; 13(1): 137-146.

32. Lau S, Lu X, Balsamo L, Devidas M, Winick $\mathrm{N}$, Hunger SP et al. Family life events in the first year of acute lymphoblastic leukemia therapy: a children's oncology group report. Pediatr Blood Cancer [Internet]. 2014 Dec [cited 2017 Oct 08]; 61(12): 2277-84. Available from: https://www.ncbi.nim.nih.gov/m/ pubmed/25175168

33. Chaparro L. Como se constituye el "vínculo especial" de cuidado entre la persona con enfermedad crónica y el cuidador familiar. Aquichan. 2011; 11(1): 7-22.

34. Ostiguín-Meléndez RM, Rivas-Herrera JC, 
Vallejo-Allende M, Crespo-Knopfler S, Alvarado-Aguilar S. Habilidades del cuidador primario de mujeres mastectomizadas. Invest. educ. enferm. 2012; 30(1): 9-17.

35. Carreño S, Chaparro L. Agrupaciones de cuidadores familiares en Colombia: perfil, habilidad de cuidado y sobrecarga. Pensamiento Psicológico [Internet]. 2017 Ene-Jun [citado
08 Oct 2017]; 15(1). Disponible en: http:// revistas.javerianacali.edu.co/index.php/pensamientopsicologico/article/view/1393

36. Mesa Melgarejo L, Carrillo Algarra AJ, Moreno Rubio F. La cronicidad y sus matices: estudio documental. Investigación en Enfermería: Imagen y Desarrollo. 2013; 15(2): 95-114. 